\title{
PREVALENCIA DE LA CIRUGÍA DE CONTROL DE DAÑOS EN PACIENTES DIAGNOSTICADOS CON TRAUMA ABDOMINAL Y TORACOABDOMINAL EN EL HOSPITAL TRAUMATOLÓGICO DR. NEY ARIAS LORA; DURANTE EL PERÍODO ENERO 2014 -DICIEMBRE 2015, SANTO DOMINGO, REPÚBLICA DOMINICANA
}

\section{Prevalence of Damage Control Surgery in patients diagnosed with abdominal and tho- racoabdominal trauma at Hospital Traumatológico Dr. Ney Arias Lora in the period January 2014 - December 2015, Santo Domingo, Dominican Republic}

\section{Dr. Alejandro Soto*, Dra. Violeta González**, Dra. Elizabeth Ramírez Báez***, Dr. Manuel de Jesús Escaño****, Dr. Miguel Mejía Sang****, Dra. Laura Guzmán Pérez****}

Recibido: 3 Marzo, 2018 Aprobado: 2 Junio, 2018

Cómo citar: Mejía Sang M, de Jesús M, Ramírez E, Soto A, Guzmán L. Prevalencia de la cirugía de control de daños en pacientes diagnosticados con trauma abdominal y toracoabdominal en el Hospital Traumatológico Dr. Ney Arias Lora; Período enero 2014-diciembre 2015, Santo Domingo, República Dominicana. Ciencia Y Salud [Internet]. 14sep.2018 [citado 14 sep.2018]; 2(3):19-6. Available from: https://revistas.intec.edu.do/index.php/cisa/article/ view/1276

\section{Resumen}

Objetivo: determinar la prevalencia de la cirugía de control de daños y el impacto sobre la recuperación de los pacientes con trauma abdominal y toracoabdominal.

Materiales y métodos: Se realizó una investigación de tipo observacional, descriptiva y transversal, con información retrospectiva, donde se revisaron los expedientes de 32 pacientes diagnosticados con trauma abdominal o toracoabdominal, a los que se les haya realizado la cirugía de control de daños en el Hospital Traumatológico Dr. Ney Arias Lora, en el período enero 2014-diciembre 2015.

Resultados y discusión: El 59\% $(\mathrm{n}=19)$ de los pacientes tenían entre 15-30 años. El 81\% $(n=26)$ eran del sexo masculino. El 53\% ( $\mathrm{n}=17)$ presentó trauma cerrado de abdomen, mientras que $44 \%(n=14)$ presentó el accidente de vehículo de motor como causa de traumatismo. En los

\footnotetext{
Miguel Mejía Sang: miguel.mejia.sang@gmail.com Manuel de Jesús: manurdejesus@gmail.com

Elizabeth Ramírez: elizabetheramirezmd@gmail.com

Alejandro Soto: alejandrosotomendez@gmail.com

Laura Guzmán: 1m.guzp@gmail.com
}

hallazgos quirúrgicos un 31\% $(\mathrm{n}=10)$ presentó la lesión hepática AAST Grado IV. El 66\% (n=21) de los casos no presentó complicaciones, y un $16 \%(\mathrm{n}=5)$ presentó alteraciones hemodinámicas. La tasa de mortalidad durante o después de la realización de dicho procedimiento fue de 0.0317 x 1000 , lo cual también puede ser expresado como proporción de mortalidad de $32 \%$.

Conclusiones: los jóvenes y adultos del sexo masculino son los más propensos a sufrir accidentes de cualquier tipo, convirtiéndolos en candidatos para la realización de cirugía de control de daños. Se infiere que la mayoría de la población estuvo expuesta a accidentes de vehículo de motor, transformándose éste, en un factor de riesgo para desarrollar trauma cerrado de abdomen.

Palabras clave: cirugía, cirugía de control de daños, trauma, trauma abdominal, trauma toracoabdominal.

\section{Abstract}

Objective: to determine the prevalence of damage control surgery and the impact on the recovery of these patients.

Material and methods: an observational, descriptive, transversal, retrospective study was carried out at the 


\section{Dr. Alejandro Soto, Dra. Violeta González, Dra. Elizabeth Ramírez Báez, Dr. Manuel de Jesús Escaño, Dr. Miguel Mejía Sang, Dra. Laura Guzmán Pérez}

Hospital Traumatológico Dr. Ney Arias Lora, information was collected from records of 32 patients diagnosed with abdominal or thoracoabdominal trauma who underwent damage control surgery in said hospital in the period January 2014 - December 2015.

Results and discussion: $59 \%(\mathrm{n}=19)$ of the patients were between $15-30$ years old. $81 \%(\mathrm{n}=26)$ were males. $53 \%$ $(\mathrm{n}=17)$ presented closed abdominal trauma, while $44 \%$ $(\mathrm{n}=14)$ presented the motor vehicle accident as the cause of trauma. In $31 \%(n=10)$ of the cases presented the surgical finding found was the AAST Grade IV liver injury. $66 \%(n=21)$ of cases presented no complications, while $16 \%(n=5)$ presented hemodynamic alterations (persistent bleeding). The mortality rate during or after the completion of this procedure was $0.0317 \times 1000$, which can also be expressed as mortality rate $32 \%$.

Conclusions: young people and adults of male sex are most likely to suffer from accidents of any kind, making them candidates for the performance of damage control surgery. It is inferred that the majority of the population was exposed to motor vehicle accidents, transforming it into a risk factor for the development of closed abdominal trauma.

Keywords: surgery, damage control surgery, trauma, abdominal trauma, thoracoabdominal trauma.

\section{Introducción}

Desde hace más de un siglo, la atención quirúrgica constituye un componente esencial de la asistencia sanitaria en todo el mundo. Dada la creciente incidencia de los traumatismos, los cánceres y las enfermedades cardiovasculares; el peso de la cirugía en los sistemas de salud públicos ha ido en aumento. Se calcula que en todo el mundo se realizan cada ańo, 234 millones de operaciones de cirugía mayor, lo que equivale a una operación por cada 25 personas. A menudo, el tratamiento quirúrgico es el único que puede mitigar discapacidades y reducir el riesgo de muerte por afecciones comunes. Se calcula que cada año 63 millones de personas se someten a intervenciones quirúrgicas por lesiones traumáticas, otros 10 millones por complicaciones relacionadas con el embarazo, y 31 millones más por problemas oncológicos ${ }^{1}$.

El trauma constituye una de las principales causas de muerte en todo el mundo, por detrás de las cardiopatías, neoplasias malignas, enfermedad cerebrovascular y neumopatías crónicas. Las principales causas de muerte por trauma son las lesiones penetrantes (heridas por proyectil de arma de fuego y por instrumento punzocortante), y por trauma contuso (accidentes en vehículos de motor, caídas $\mathrm{y}$ atropellamiento), con una gran incidencia en sujetos menores de 30 años $^{2}$.

La cirugía de control de daños es una técnica quirúrgica que intenta evitar la tríada letal de coagulopatía, hipotermia y acidosis en los pacientes con múltiples lesiones. Consiste en tres fases: laparotomía inicial, estabilización en Unidad de Cuidados Intensivos (UCI) y cirugía definitiva. Durante la laparotomía inicial se realizan esfuerzos para controlar el sangrado quirúrgico y no quirúrgico (mediante empaquetamiento), y evitar la contaminación peritoneal. En la fase de estabilización se corrigen la hipotermia, la acidosis y la coagulopatía en la UCI. Durante la cirugía definitiva se retira el empaquetamiento y se realiza la reparación definitiva de las lesiones ${ }^{2}$.

En nuestro estudio, el interés está enfocado en identificar la prevalencia de la cirugía de control de daños, el cual se llevó a cabo utilizando los casos reportados al Departamento de Epidemiología del Hospital Traumatológico Dr. Ney Arias Lora, durante el período de tiempo enero 2014 - diciembre de 2015. La importancia de este estudio radica en poder demostrar qué tan frecuente es este tipo de procedimientos y el impacto que tiene sobre la recuperación de los pacientes, identificando entonces cuáles son las posibles complicaciones, grupo etario más afectado, los hallazgos quirúrgicos más frecuentes y su tasa de mortalidad; con el fin de brindar una mejor calidad de atención a estos pacientes. 


\section{Material y métodos}

Se llevó a cabo un estudio observacional, descriptivo de tipo transversal, utilizando los expedientes de los pacientes con trauma abdominal y toracoabdominal, a los que se le realizó la cirugía de control de daños en el Hospital Traumatológico Dr. Ney Arias Lora, entre enero del 2014 y diciembre del 2015, donde se evaluó la prevalencia de este procedimiento, junto con los resultados posteriores a la realización del mismo. Se utilizó el método analítico para la recolección, procesamiento e interpretación de los datos obtenidos de los archivos del hospital.

Para la recolección de los datos se utilizó un cuestionario dirigido en el que se identificó: edad, sexo, indicación preoperatoria, mecanismo de trauma, causa del traumatismo, hallazgos quirúrgicos, procedimiento realizado, complicaciones, y si hubo defunción.

Antes de utilizar dicho instrumento se realizó un plan piloto con la finalidad de validar la fiabilidad y validez del método a utilizar. Asimismo, un consentimiento informado fue pedido para la recolección, análisis, almacenamiento y reutilización de la información al centro de salud seleccionado. De la misma manera, se respetó la confidencialidad de los pacientes mediante la utilización de códigos numéricos para sus respectivos expedientes. Los datos obtenidos fueron procesados estadísticamente utilizando el programa Epi Info ${ }^{\mathrm{TM}} 7$ para Windows.

\section{Resultados}

Según los resultados obtenidos de la investigación, puede apreciarse en la Gráfica 1 la prevalencia de la cirugía de control, de daños en los pacientes con trauma abdominal o toracoabdominal.

En esta primera gráfica podemos observar que, de 189 casos de pacientes con trauma abdominal o toracoabdominal, 34 (17\%) fueron sometidos a cirugía de control de daños.

\section{Gráfica 1: Prevalencia de la cirugía de control de dańos $(\mathrm{N}=189)$}

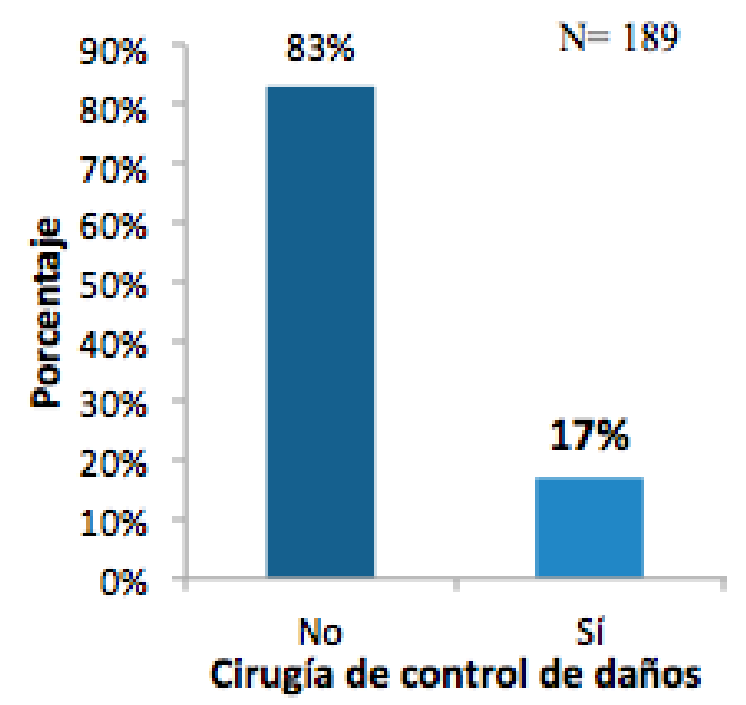

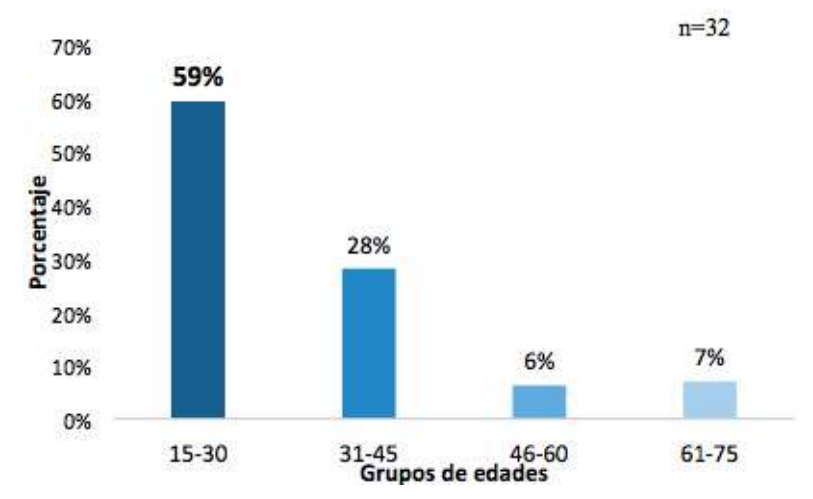

El 59\% ( $\mathrm{n}=19)$ de los pacientes estuvo en el rango de edad entre 15-30 ańos, con una media de $\chi=30.78$ y una desviación estándar de $\sigma=13,6810$. Mientras que 28\% ( $\mathrm{n}=9)$ corresponden al grupo etario de 31-45 ańos, $6 \%(\mathrm{n}=2)$ se encuentran en el grupo de 46-60 años, y 7\% $(n=2)$ pertenecen al grupo de 61-75 años. 
Gráfica 3: Distribución de acuerdo al sexo de pacientes con trauma abdominal y toracoabdominal y que se les realiza la CCD $(\mathrm{N}=32)$

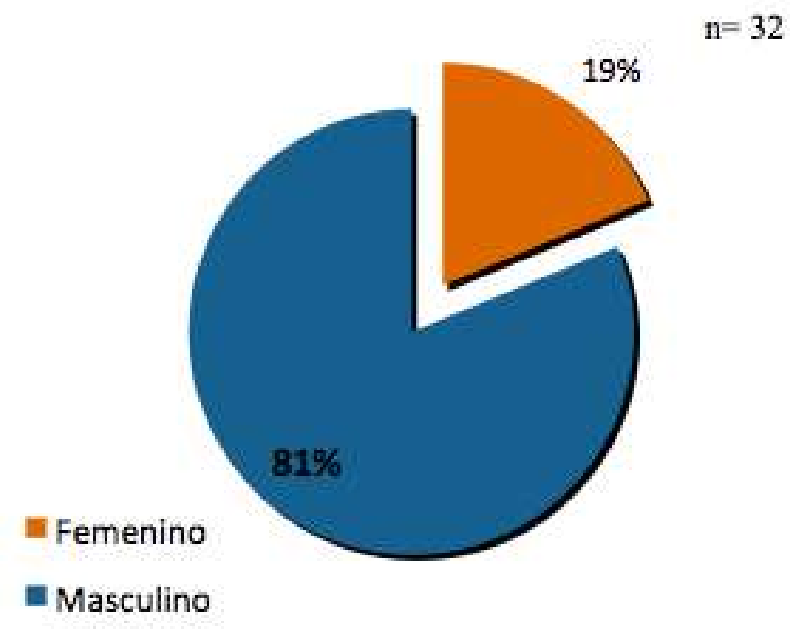

El $81 \%(\mathrm{n}=26)$ de los pacientes correspondieron al sexo masculino, y $19 \%(\mathrm{n}=8)$ al sexo femenino.

Gráfica 4: Causas de traumatismos en pacientes con trauma abdominal y toracoabdominal que se les realiza la $\mathrm{CCD}(\mathrm{N}=32)$

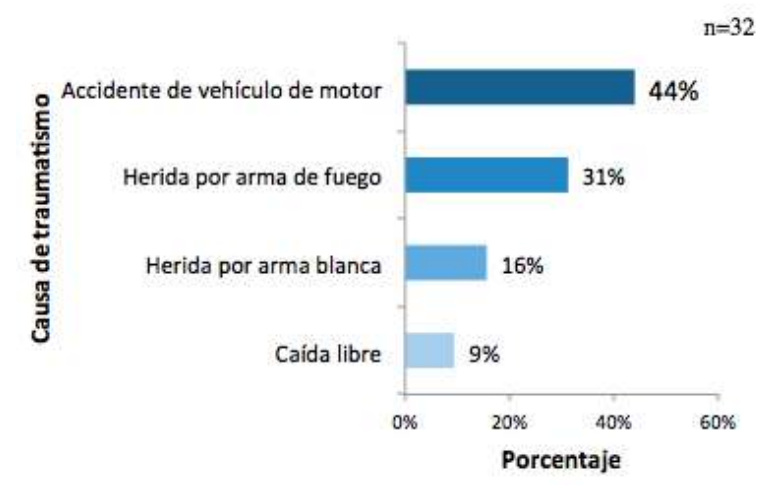

Como causa de traumatismo, el $44 \%(\mathrm{n}=14)$ presentó el accidente de vehículo de motor, el 31\% $(n=10)$ herida por arma de fuego, el $16 \%(n=5)$ herida por arma blanca y $9 \%(\mathrm{n}=3)$ presentó caída libre.
Gráfica 5: Hallazgos quirúrgicos en pacientes con trauma abdominal y toracoabdominal que se les realiza la cirugía de control de daños $(\mathrm{N}=32)$

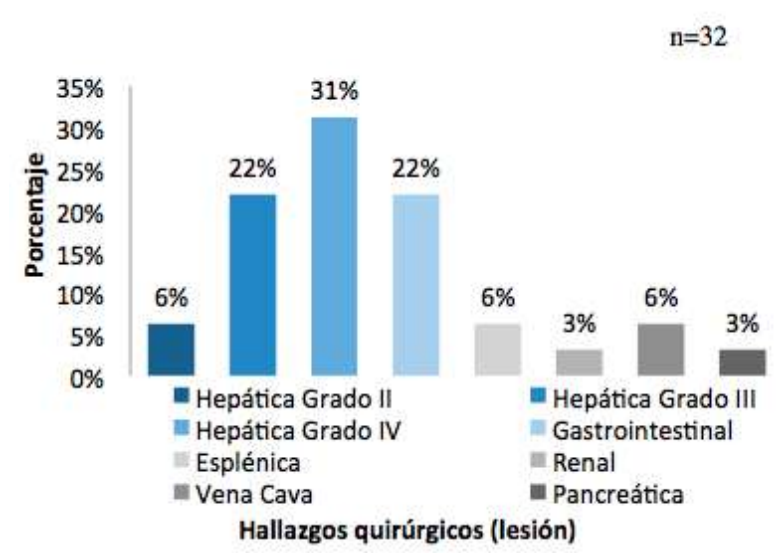

Entre los hallazgos quirúrgicos que presentaron encontramos: $31 \%(\mathrm{n}=10)$ con lesión hepática AAST Grado IV, un 22\% $(\mathrm{n}=7)$ lesión hepática AAST Grado III, un 6\% $(\mathrm{n}=2)$ lesión hepática AAST Grado II, otro $22 \%(\mathrm{n}=7)$ lesión gastrointestinal, un $6 \%(n=2)$ lesión esplénica, otro $6 \%(n=2)$ lesión de la vena cava, un $3 \%(\mathrm{n}=1)$ lesión renal, y otro $3 \%(\mathrm{n}=1)$ lesión pancreática.

Gráfica 6: Complicaciones en pacientes con trauma abdominal y toracoabdominal que se les realiza la cirugía control de daños $(\mathrm{N}=32)$

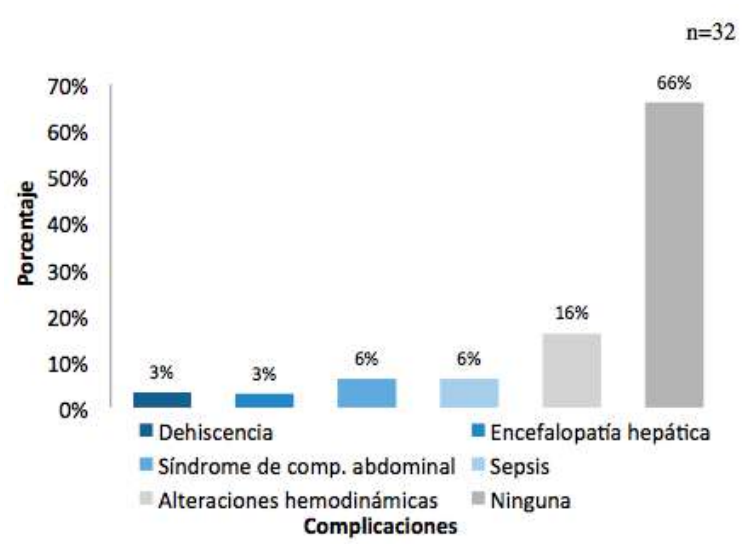

El 66\% $(n=21)$ de los casos no presentaron complicaciones, mientras que un $34 \%$ sí presentaron, 
entre las que se encuentran: $16 \%(\mathrm{n}=5)$ alteraciones hemodinámicas (hemorragia persistente), un 6\% $(\mathrm{n}=2)$ síndrome de compartimiento abdominal, otro $6 \%(n=2)$ sepsis, un $3 \%(n=1)$ encefalopatía hepática, y otro $3 \%(\mathrm{n}=1)$ dehiscencia.

Gráfica 7: Distribución de mortalidad en pacientes con trauma abdominal y toracoabdominal que se les realiza la cirugía de control de daños $(\mathrm{N}=32)$

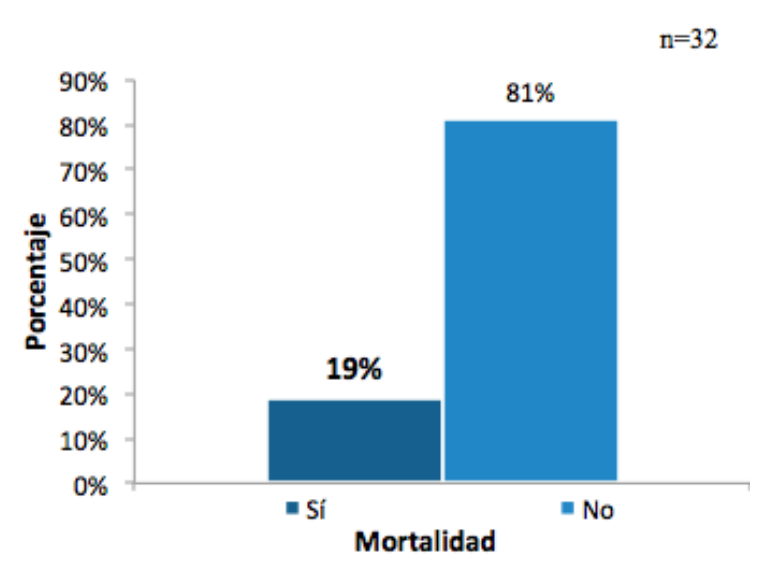

En la Gráfica 7 se evidencia la tasa de mortalidad evidenciada en el estudio, que fue de $19 \%$, correspondiendo a 6 casos.

\section{Discusión y conclusiones}

Se estudiaron 32 pacientes con trauma abdominal o toracoabdominal sometidos a la cirugía de control de daños en el Hospital Traumatológico Dr. Ney Arias Lora durante el período enero 2014 - diciembre 2015, donde se encontró una prevalencia de pacientes a los que se les realizó el procedimiento de $17 \%$.

Dentro de las características sociodemográficas, el sexo más común fue el masculino, representando el $81.25 \%$ de los casos. Estos datos son similares a los planteados por Vyhnánek et $\mathrm{al}^{3}$. en su estudio, donde el sexo más frecuente fue el masculino con un $81 \%$, y un $19 \%$ correspondiente al sexo femenino. Este predominio masculino se debe a que en la mayoría de los casos es quien se responsabiliza de las tareas fuera del hogar y se expone a múltiples accidentes de tránsito, que en nuestro país cada día van en aumento. Estos datos se ven respaldados por el reporte ofrecido por el Observatorio de Seguridad Ciudadana de República Dominicana en su boletín estadístico de enero a junio del 2015, en donde se plantea que el sexo predominante en los accidentes de tránsito es el masculino ${ }^{4}$.

Igualmente, determinamos que el rango de edad más común entre los pacientes a los que se les realiza la Cirugía de control de daños fue de 15-30 ańos, con un 59\%, una media de $\chi=30.78$ y una desviación estándar de $\sigma=13,6810$. El rango de edad de $31-46$ obtuvo un $28 \%$, seguido del rango de edad de 47-61, el cual obtuvo un 6\%; y por último, los mayores de 62 años que formaron un 7\% de los casos. Estos datos comparten similitud con los del estudio de Sharp et al..$^{5}$ quienes concluyeron que la edad media de los integrantes de su muestra fue de 33 años. Estos resultados pueden ser atribuibles al hecho de que los jóvenes son la población más expuesta a la violencia y más propensa a sufrir de accidentes, lo cual podría ser ocasionado por su movilidad y su inmadurez. Estos datos son similares a los ofrecidos por el Observatorio de Seguridad Ciudadana de República Dominicana en su boletín estadístico de enero-junio del 2015 donde la edad predominante por accidentes de tránsito correspondió a los pacientes entre los 20 y 24 años de edad, seguido del grupo de 25 a 29 años .

Los datos antes expuestos nos sugieren entonces que los jóvenes y adultos jóvenes del sexo masculino son los más propensos a sufrir accidentes de cualquier tipo, lo cual los convierte en candidatos para la realización de la cirugía de control de daños. Se estima que para el año 2030 hayan alrededor de 43,811 muertes por accidentes de vehículos de motor, si no se toman las medidas de lugar tanto a nivel preventivo como a nivel resolutivo ${ }^{6}$. Esto es sin contar las personas discapacitadas que podrían resultar de estos accidentes. 


\section{Dr. Alejandro Soto, Dra. Violeta González, Dra. Elizabeth Ramírez Báez, Dr. Manuel de Jesús Escaño, Dr. Miguel Mejía Sang, Dra. Laura Guzmán Pérez}

De este mismo modo, al buscar conocer la causa de traumatismo más frecuente encontramos que el accidente de vehículo de motor fue la más vista con un $43.7 \%$, seguido de heridas por arma de fuego, en un $33 \%$, heridas de arma blanca en un $16 \%$, y caída libre en un $9 \%$ de los casos. Nuestros datos de frecuencia se asemejan al estudio de Doklestic et al. ${ }^{7}$; donde los accidentes de vehículo de motor fueron la principal causa de trauma abdominal, encontrado en el $66.1 \%$ de los pacientes. Esto podría ser atribuible a que según las estadísticas de la Organización Mundial de la salud (OMS), la República Dominicana es el primer país del continente americano con más muertes de tránsito, por cada 100 mil habitantes con una tasa de 29.3 fallecimientos ${ }^{8}$. Según el reporte realizado por el Observatorio de Seguridad Ciudadana en su boletín estadístico de enero-junio del 2015, se concluyó que durante los primeros 6 meses del ańo la mayoría de las muertes violentas fueron ocasionadas por accidentes de tránsito ${ }^{4}$.

Por tanto, podemos inferir que la mayor parte de la población estuvo expuesta a accidentes de vehículo, convirtiéndose éste en un factor de riesgo para desarrollar trauma cerrado de abdomen. Según la Organización mundial de la Salud (2010) las defunciones causadas por los traumatismos son un problema en aumento. $S$ prevé que las tres causas principales de defunción por traumatismos a nivel mundial son las colisiones en las vías de tránsito, homicidio y suicidio, las cuales, pasarán por delante de otras causas de defunción y figurarán entre las 20 principales causas de muerte en el mundo en el $2030^{6}$.

Otro objetivo para nuestra investigación fue el hallazgo quirúrgico más encontrado, que fue la lesión hepática AAST grado IV, con un 31\%; la lesión hepática AAST grado III, en un $23 \%$; lesiones gastrointestinales en un $22 \%$, lesiones hepáticas AAST grado II, lesión de vena cava y lesión esplénica en un $6 \%$ de los casos, cada uno; y lesión renal y pancreática en un 3\% de los casos, cada uno. Este resultado es similar al encontrado por Doklestic et al. ${ }^{7}$ donde el hallazgo quirúrgico más común fue la lesión hepática AAST grado IV, correspondiente a un $43.8 \%$ de los casos. A pesar de contar con una protección anatómica, el hígado es el órgano más lesionado en los traumas abdominales. Esto es debido a que, en el trauma cerrado de abdomen, las vísceras afectadas en su mayoría son las vísceras sólidas, mientras que, en las lesiones de tipo penetrante, las vísceras afectadas son las huecas?.

Es pertinente concluir entonces que las lesiones hepáticas de alto grado son las más frecuentes, lo cual se debe a la naturaleza de los traumatismos que hemos encontrado con mayor frecuencia en nuestro estudio.

Al indagar acerca de las complicaciones más frecuentes dimos con el resultado que no hubo complicaciones reportadas en el 66\% de los casos. En los casos que sí presentaron complicaciones, la más común fueron las alteraciones hemodinámicas, presentes en un $16 \%$, seguido de síndrome de compartimiento abdominal y sepsis en un $6 \%$ cada uno, y dehiscencia de herida y encefalopatía hepática en un 3\% cada uno. Este resultado es equiparable al del estudio de Sharp et $\mathrm{al}^{5}$. donde el $23 \%$ de la población presentó el shock hipovolémico como complicación. Cuando ocurre la coagulopatía, los intentos quirúrgicos para controlar el tipo de sangrado no quirúrgicos, especialmente del hígado y retroperitoneo, con frecuencia no tienen éxito. ${ }^{10}$

Es de conocimiento universal que el hígado tiene un suministro sanguíneo de $1.500 \mathrm{ml} / \mathrm{min}$, además de que es el sitio principal de la síntesis de todos los factores de la coagulación, a excepción del factor VIII. Por lo tanto, la gestión operativa adecuada de una lesión hepática importante es un componente clave para una laparotomía de control de daños exitosa. Nuestro estudio arrojó como resultado, que el hallazgo quirúrgico más encontrado fue la lesión hepática AAST grado IV; en este tipo de lesiones $\mathrm{y}$ en las lesiones grado III, el procedimiento más 
utilizado es el empaquetamiento hepático. Esta técnica, aunque muy provechosa, tiene sus riesgos, y uno de ellos es un $22 \%$ de probabilidad de resangrado, según datos expuestos en el estudio de Parks et $\mathrm{al}^{11}$.

Las alteraciones hemodinámicas (hemorragia persistente) fue la complicación más frecuente presentada en los pacientes de nuestro estudio, por lo que es recomendable llevar pleno control de los signos de shock, que podría presentar el paciente al momento de la realización del procedimiento, de manera que se pueda detectar y tratar agudamente. Recalcando que Sharp et al..$^{5}$ obtuvieron en su estudio que, de todos los pacientes que fueron sometidos a empaquetamiento intra-abdominal, en el 23\% de ellos permaneció la hemorragia.

Se evidenció una proporción de mortalidad de $19 \%$, dato parecido al encontrado previamente por el estudio de Timmermans et al. ${ }^{12}$ que fue de un $27 \%$ de mortalidad global. Otro estudio de Hatch et al. ${ }^{13}$ también tiene como resultado un $27 \%$ en cuanto a la mortalidad global, mientras que en la población del estudio de Sharp et al. el porcentaje se duplicó con un $44 \%$ de mortalidad global. La mayoría de los pacientes con trauma grave que sobreviven son jóvenes, y éstos afrontan una mejor expectativa vital que los pacientes no tan jóvenes.

\section{Referencias}

1. Organización Mundial de la Salud. La cirugía segura salva vidas. [internet]. 2008 [Citado 2015 agos 11] Disponible en http://www. who.int/patientsafety/safesurgery/sssl_brochure_spanish.pdf

2. Camacho J, Mascareño S. Gaceta Médica Cirugía de Control de Daños. Gaceta Médica de México [internet]. 2013 [citado en 2015 agos 12];149(6):61-72. Disponible en http://www.anmm.org.mx/GMM/2013/n1/ GMM_149_2013_1_061-072.pdf
3. Vyhnánek F, Duchác V, Skála P. Damage control laparotomy in blunt abdominal injury. Acta Chir Orthop Traumatol Cech. 2009 Aug;76(4):310-3.

4. Observatorio de Seguridad Nacional, Republica Dominicana Informe Estadístico sobre Seguridad Ciudadana OSC-IE 016 [internet]. 2015 [Citado 2016 feb 16]. Disponible en http://www.mip.gob.do/images/docs/Programas/Boletines/Boletn_Enero_ Junio_2015.pdf

5. Sharp k, Locicero R. Abdominal packing for surgically uncontrollable hemorrhage. Ann Surg. 1992;215(5):467-475.

6. Organización Mundial de la Salud. Traumatismos y Violencia [internet]. Ginebra: 2015 [citado en 2015 agos 12]. Disponible en http://whqlibdoc.who.int/publications/2010/9789243599373_spa.pdf

7. Doklestic K, Stefanovic B, Gregoric P, Ivancevic $\mathrm{N}$, et al. Surgical management of AAST grades III-V hepatic trauma by Damage control surgery with perihepatic packing and definitive hepatic repair-single centre experience. World J Emg Surgery. 2015 Aug;10:34. doi: 10.1186/s13017-015-0031-8.

8. Organización Mundial de la Salud.Global Status Report On Road Safety [internet] 2015 [Citado 2016 feb 21] Disponible en http:// www.who.int/violence_injury_prevention/ road_safety_status/2013/report/es/

9. Hospital Traumatológico Dr. Ney Arias Lora [internet] 2016 [consultado en 2015 agos 12]. Disponible en http://www.hospitalneyarias. gov.do/index.html

10. AhmedN, Jerome JV, Management of Liver Trauma in Adults. Journal of Emergencies, Trauma and Shock. 2011 Jan;4(1):1;114-119 
11. Parks RW, Chrysos E, and Diamond T. Management of liver trauma. B J Surg. 1999; 86:1121-1135.

12. Timmermans J, Nicol A, Kairinos N, Teijink J, Prins M, Navsaria P. Predicting mortality in damage control surgery for major abdominal trauma. S Afr J Surg. 2010 Feb;48(1):6-9.

13. Hatch QM, Osterhout LM, Podbielski J, Kozar RA, Wade CE, Holcomb JB, Cotton BA. Impact of closure at the first take back: complication burden and potential overutilization of damage control laparotomy. J Trauma. 2011 Dec;71(6):1503-11.

14. WHO. Dominican Republic: WHO statistical profile. Country statistics and global health estimates by $\mathrm{WHO}$ and UN partnerts. [Retrieved 2015 Jul 25] Available from http:// who.int/gho/mortality_burden_disease/en/

15. Germanos S, Gourgiotis S, Villias C, Bertucci M, Dimopoulos N, Salemis, N Damage control surgery in the abdomen: An approach for the management of severe injured patients. International Journal of Surgery. 2007 Jun;6: 246-252.

16. Pontificea Universidad Católica de Chile Primera sección. Paotología traumática. Politraumatizado. Escuela de Medicina PUC. (s.f) [Citado 2015 agos 12] Disponible en http://escuela.med.puc.cl/paginas/publicaciones/TextoTraumatologia/Trau_Secc01/Trau_ Sec01_56.html

17. Fonseca JC, González JC, Frometa A, Fonseca T. Prognostic factors of death in patients with multiple traumas. [internet]. 2007 [cited 2015 Agos 12]. Disponible en http://bvs.sld.cu/revistas/mie/vol10_3_11/mie03311.html

18. Pueo F, Rojas F, Rosset G, Rovey L, Sanchez MS. Mecanismo de los Choques automóviles. [internet] 2012 [Citado $2016 \mathrm{Feb}$ 16]. Disponible en http://www.reeme.arizona. edu/materials/El\%20mecanismo $\% 20 \mathrm{de} \% 20$ los $\% 20$ choques.pdf 